\title{
An Optimal Real-Time Controller for Vertical Plasma Stabilization
}

\author{
N. Cruz, J.-M. Moret, S. Coda, B. P. Duval, H. B. Le, A. P. Rodrigues, C. A. F. Varandas, C. M. B. A. Correia, and \\ B. Gonçalves
}

\begin{abstract}
Modern Tokamaks have evolved from the initial axisymmetric circular plasma shape to an elongated axisymmetric plasma shape that improves the energy confinement, which is one of the key factors to improve fusion reactor performance. However, the elongated plasma cross section introduces a vertical instability that requires a real-time feedback control loop to stabilize the plasma vertical position and velocity. At the Tokamak à Configuration Variable (TCV) in-vessel poloidal field coils driven by fast switching power supplies are used to stabilize highly elongated plasmas. TCV plasma experiments have used a PID algorithm based controller to correct the plasma vertical position. In late 2013 experiments a new optimal real-time controller was tested improving the stability of the plasma. This contribution describes the new optimal real-time controller developed. The choice of the model that describes the plasma response to the actuators is discussed. The high order model that is initially implemented needs the application of a mathematical order reduction and the validation of the new reduced model. The lower order model is used to derive the time optimal control law. A new method for the construction of the switching curves of a bang-bang controller is presented that is based on the state-space trajectories that optimize the time to target of the system. The final control algorithm and its implementation are described and preliminary experimental results are discussed.
\end{abstract}

Index Terms-Optimal control, plasma control, real-time, tokamak.

\section{INTRODUCTION}

$\mathbf{M}$ ODERN tokamak devices [1] are designed to accommodate elongated cross-section plasmas [2][3] to improve fusion performance. A vertically elongated plasma

\footnotetext{
Manuscript received June 16, 2014; revised December 12, 2014; accepted September 08, 2015. Date of publication October 29, 2015; date of current version December 11,2015. This work was supported in part by EURATOM and carried out within the framework of the European Fusion Development Agreement, in part by the "Fundação para a Ciência e Tecnologia" through project Pest-OE/SADG/LA0010/2013, and in part by the Swiss National Science Foundation. The views and opinions expressed herein do not necessarily reflect those of the European Commission.

N. Cruz is with the Instituto de Plasmas e Fusão Nuclear, Instituto Superior Técnico, Universidade de Lisboa, P-1049-001 Lisboa, Portugal (e-mail: nunocruz@ipfn.ist.utl.pt).

J.-M. Moret, S. Coda, B. P. Duval, and H. B. Le are with the Centre de Recherches en Physique des Plasmas, École Polytechnique Fédérale de Lausanne (EPFL), CH-1015 Lausanne, Switzerland.

A. P. Rodrigues, C. A. F. Varandas, and B. S. Gonçalves are with the Instituto de Plasmas e Fusão Nuclear, Instituto Superior Técnico, Universidade de Lisboa, P-1049-001 Lisboa, Portugal.

C. M. B. A. Correia is with the Centro de Instrumentação, Departamento de Física, Universidade de Coimbra, P-3004-516 Coimbra, Portugal.

Color versions of one or more of the figures in this paper are available online at http://ieeexplore.ieee.org.

Digital Object Identifier 10.1109/TNS.2015.2478965
}

presents important advantages since it allows the creation of divertor plasmas, the increase of density limit as well as providing plasma stability. However, an elongated plasma is unstable due to the forces that pull the plasma column upward or downward. The result of these forces is a plasma configuration that tends to be pushed up or down depending on the initial displacement disturbance. For example, a small displacement downwards results in the lower poloidal field coils pulling the plasma down, with increased strength as the plasma gets further from the equilibrium position. To compensate this instability, feedback controllers have been designed to correct the vertical position displacement [4]-[6].

The design of vertical stabilization feedback controllers has been based on simple models, resulting in experimentally tuned Single Input Single Output (SISO) Proportional Integral and Derivative (PID) regulators. This procedure requires an in-depth experimental treatment that is time consuming and requires a big number of experimental discharges to obtain the necessary gains optimization. Optimization principles have been used to design high performance controllers for the vertical stabilization of the ITER plasma column [7][8]. This paper presents an alternative method to design the vertical stabilization controller of a tokamak using a simple plasma model and the application of optimal control theory.

This paper is organized as follows: Section II presents the vertical observer developed to detect the plasma centroid vertical position and velocity in real-time; Section III briefly depicts the different methods that can be used to describe a tokamak plasma; Section IV describes the state-space plasma model that predicts the plasma response to the actuators and the model reduction performed to allow the application of the time optimal control theory that is presented in Section V; Section VI depicts the simulation tool that grants off line testing and parameter tuning of the controller; The controller results and future work is presented in Section VII.

\section{Vertical Plasma Position Observer}

The vertical position observer uses a linear combination of magnetic field probe signals. The matrix coefficients of the probes signals are calculated before each plasma discharge, accounting for plasma parameters such as shape and position. This means that, although the real-time vertical position observer is independent of these plasma parameters, the contribution of each probe to the observer has in account the pre-planned plasma parameters. For example, when the experiment aims at creating a plasma in the upper part of the vacuum vessel, the probes closer to the predicted plasma will be more efficient 
estimating its position, thus being given more weight in the observer.

The coefficients calculated are the $z I_{p}$ observer. From a finite element set of plasma current filaments, using Green's functions, the magnetic field produced in the probes by the filaments is calculated. A-matrix is built with the probes that are going to be used, which are inverted to obtain the observer coefficients [9].

The plasma current density is decomposed into $m_{h}$ by $n_{h}$ vertically bilinear finite elements evenly distributed in a two dimensional poloidal cut of the vessel. This defines a rectangle with boundaries $[a, b] \times[c, d]$ set to include the plasma inside it according to the pre programmed equilibria defined in the discharge preparation, storing its values in the matrix $T_{x h}$ according to [10]:

$$
\begin{aligned}
h_{i j}(r, z) & =\left(1-\frac{\left|r-r_{i}\right|}{\Delta r}\right)\left(1-\frac{\left|z-z_{j}\right|}{\Delta z}\right)\left[\left|r-r_{i}\right|\right. \\
& \leq \Delta r]\left[\left|z-z_{j}\right| \leq \Delta z\right], \\
i & =1 \ldots m_{h}, j=1 \ldots n_{h}
\end{aligned}
$$

where

$$
\begin{aligned}
\Delta r & =\frac{b-a}{m_{h}+1}, \Delta z=\frac{d-c}{n_{h}+1}, \\
r_{i} & =a+i \Delta r, z_{j}=c+j \Delta z
\end{aligned}
$$

and the square brackets represent the Iverson brackets with value 1 for a true condition and 0 otherwise. The current density can thus be written as a linear combination with coefficients $a_{h}[10]$ :

$$
j_{\varphi}\left(r_{x}, z_{x}\right) \Delta r \Delta z=I_{x}=T_{x h} \cdot a_{h}
$$

Using this geometry and introducing the weights $w_{m}$ that allow giving more relevance to some measures over others, the magnetic field probe measurements can be related to the current density by:

$$
w_{m} B_{m}=w_{m}\left(B_{m c} I_{c}+B_{m x} T_{x h} a_{h}\right)
$$

where $B_{m}$ is the vector of the measured quantities in the magnetic probes, $B_{m c}$ is the matrix of the Green functions between the coils and the magnetic probes, $I_{c}$ is the vector of the poloidal field coil currents, $B_{m x}$ is the matrix with the Green functions to link the current in the plasma filaments with the magnetic field measured by the probes.

The weighted coefficients can be obtained taking the term with the current density from equation (3) and solving in order to $a_{h}$ :

$$
a_{h}=A_{h}^{-1}\left(A_{h T} w_{m} B_{m}-A_{h T} w_{m} B_{m c} I_{c}\right)
$$

with $A_{h}$ and $A_{h T}$ given by:

$$
\begin{aligned}
A_{h} & =T_{x h}^{T} B_{m x}^{T} w_{m}^{T} w_{m} B_{m x} T_{x h} \\
A_{h T} & =T_{x h}^{T} B_{m x}^{T} w_{m}^{T}
\end{aligned}
$$

Using the Einstein notation and replacing $I_{x}$ according to 2, the estimation of the plasma vertical position, the so-called observer, is defined by the static relation [11]:

$$
z I_{p}=\left(z_{x}^{T}-z_{a}\right) \cdot I_{x}=Z_{x}^{T} A_{m} B_{m}-Z_{x}^{T} A_{c} I_{c}
$$

where $\mathrm{z}$ is the position of the plasma column, $z_{x}$ is the position of the filaments of the plasma column and $z_{a}$ is the reference position of the plasma axis. $Z_{x}$ is the vector of relative positions given by $Z_{x}=z_{x}-z_{a} . A_{m}$ and $A_{c}$ are given by:

$$
\begin{aligned}
A_{m} & =T_{x h} A_{h}^{-1} A_{h T} w_{m} \\
A_{c} & =T_{x h} A_{h}^{-1} A_{h T} w_{m} B_{m c}
\end{aligned}
$$

The plasma velocity observer $\left(d\left(z I_{p}\right) / d t\right)$ uses the same method. Since the time derivative of $I_{c}$ has slow variation compared to vertical position growth rate, it is not relevant for the control system. In consequence, the following reduced equations define the Bdot observer:

$$
\begin{aligned}
\frac{d I_{x}}{d t} & =A_{m} \cdot \frac{d B_{m}}{d t} \\
\frac{d\left(z I_{p}\right)}{d t} & =Z_{x}^{T} \frac{d I_{x}}{d t}
\end{aligned}
$$

\section{PlASMA DESCRIPTION}

The modeling of a tokamak plasma requires complex mathematical calculation, in depth physical knowledge and computational power for numerical calculation during simulation phase. Different paths have been tried to accomplish this mission.

The simpler models consider the plasma as a filament or nondeformable matrix of conducting filaments. The more complex models include nonlinear codes, with the simulation of nonlinear behaviors such as large vertical position displacements. Some important plasma model and reconstruction codes include PET[12], TSC (Tokamak Simulation Code)[13], EFIT (Equilibrium FITting)[14], FBT (Free Boundary Tokamak)[15], $P R O$ TEUS[16], CREATE-L[17], DINA[18] and RZIP [19].

Some of these codes are accurate for plasma simulation and reconstruction but due to its complex structure are not suitable for controller design. This action is based on simpler linear models that ensure the stability, robustness and performance of the controller, provided that the states are not too far from equilibrium.

Linear models for control design purposes use the electrical circuit equations to calculate the time evolution of the plasma current. RZIP is an enhanced non deformable model that may vary its vertical and radial position, as well as its total plasma current. The RZIP model is presented in the next section, to be used for the design of the plasma stabilization controller.

\section{Plasma Model for Control}

\section{A. RZIP Plasma Model}

The use of the RZIP plasma model aims at finding the transfer function between the currents in the poloidal field coils, internal 
to the TCV structure close to the plasma, and the vertical plasma displacement [20][21].

The RZIP model gets its name from the simplifications assumed to build the circuit equations, with the following characteristics: (i) the current has constant distribution, rigid model, as the plasma shape is assumed not to change; (ii) the vertical position can change: plasma is free to move vertically; (iii) the radial position can change: plasma is free to move radially; (iv) the integral of the plasma filaments current can change: the total plasma current is free to change.

The model design simplifications give important advantages over more complex plasma models, maintaining an overall accuracy: (i) A simple model that is easier to implement; (ii) No need to calculate the complete plasma equilibrium; (iii) More explicit model to the quantities that define plasma response to the control variables (a better control model).

The model is derived from (i) the equilibrium equation of the vertical forces in the plasma; (ii) the equilibrium equation of the radial forces in the plasma and (iii) the plasma current circuit equations [19].

\section{B. Influence of the Voltage in the Fast Coils in the Plasma Vertical Position}

This section aims at obtaining the independent transfer function that describes the influence of the poloidal field coils internal to the TCV structure close to the plasma on the plasma vertical position.

The state space system is diagonalized to obtain the independent influence of the coil currents over the plasma vertical position. Then the equation of the fast coil is taken by neglecting the influence of the other coils. The typical way to address the vertical stabilization problem is to independently control the vertical plasma position from the plasma current and shape controllers [2], which are designed on the basis that the system is vertically stable due to the controller already implemented. This double loop arrangement simplifies the design of the controllers, based on the assumption and later confirmation that the controllers act on different time scales. Different frequencies in the controllers grant the treatment of some parameters as disturbances to the next stage of the global controller.

The verification of the method was achieved by comparing the value of the plasma vertical instability growth rates for different plasmas with the transfer function unstable pole. Fig. 1 permits to immediately check that higher elongation correspond to higher instability. Values for some discharges were validated with the growth rate measurements in TCV [21].

\section{Model Reduction and Validation}

In control engineering, the best model is not always the most accurate, but the one that allows the construction of a robust stable controller, according to the necessary performance and specifications.

For the purpose of applying optimal control theory to the plasma model obtained a model reduction was necessary to implementation of the mathematical treatment presented in the next section. The transfer function that was obtained is of 52nd order, while optimal control theory is usually applied to systems
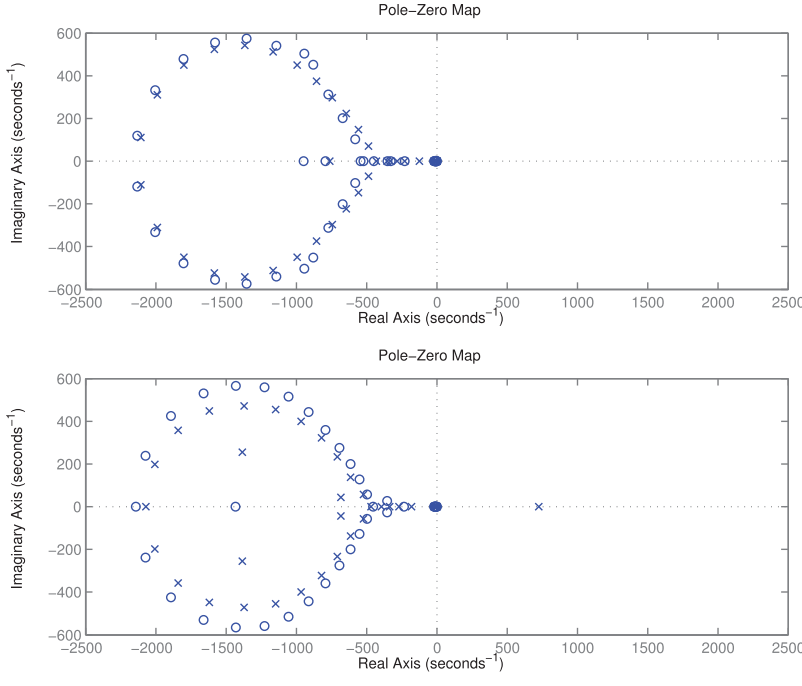

Pole-Zero Map

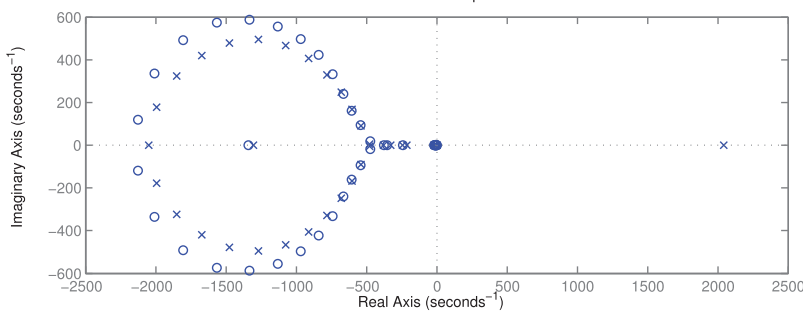

Fig. 1. Pole-Zero plot of 52nd order transfer function of a circular TCV plasma (top) standard elongated plasma (middle) and extremely elongated plasma. The poles are plotted as x's and the zeros as o's.
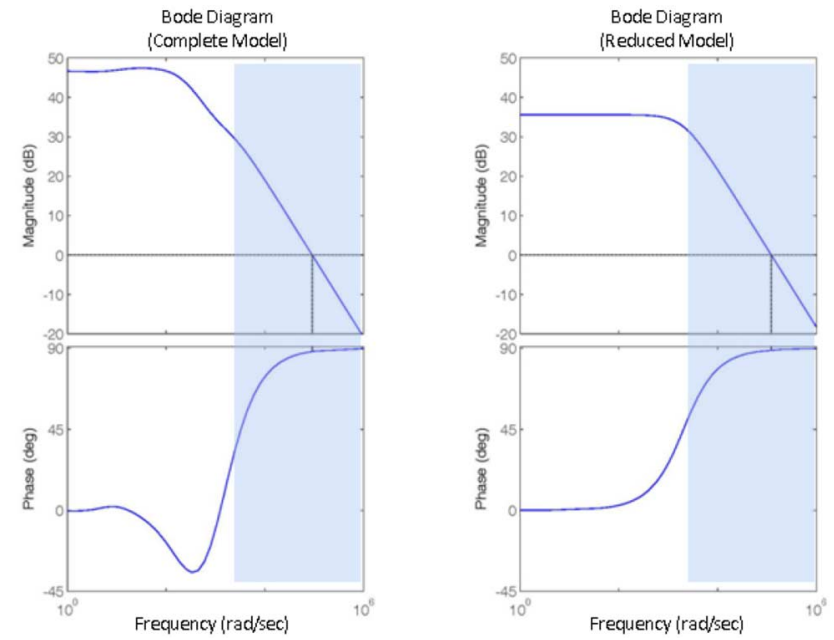

Fig. 2. Bode diagrams of the complete and reduced model transfer functions.

with second or third order at most. This led to the application of model reduction techniques, which favor obtaining a reduced system that may be solved more efficiently for control design purposes.

The method of balanced realization was applied to reduce the transfer function [22] to a second order transfer function with difference results that could not be detected by the plot of the step response of both transfer functions. In the bode diagram plot of both models (Fig. 2) differences were detected but only on slower frequencies that are not relevant for plasma vertical stabilization. The blue shadow areas in the figure show the agreement between both models for the frequencies of interest. 


\section{Optimal CONTROL}

\section{A. System Definition}

This section describes the application of the time optimal control to the second order model to obtain a control law, the switching time and the final time of the bang-bang controller [23][24].

The second order transfer-function that describes the plasma model has the form:

$$
\frac{X_{s}(s)}{U(s)}=\frac{n_{1} s+n_{2}}{s^{2}+d_{1} s+d_{2}}
$$

This transfer function represents the following controllable state space model:

$$
\dot{X}=A X+B u
$$

with $X=\left[\begin{array}{l}x_{1} \\ x_{2}\end{array}\right], A=\left[\begin{array}{cc}0 & 1 \\ -d_{2} & -d_{1}\end{array}\right], B=\left[\begin{array}{l}b_{1} \\ b_{2}\end{array}\right]$, where

$$
x_{1}=x_{s}
$$

and

$$
x_{2}=x_{1}+b_{1} u
$$

are the system variables when $b_{i}$ is given by

$$
\left[\begin{array}{c}
0 \\
n_{1} \\
n_{2}
\end{array}\right]=\left[\begin{array}{ccc}
1 & 0 & 0 \\
d_{1} & 1 & 0 \\
d_{2} & d_{1} & 1
\end{array}\right]\left[\begin{array}{l}
b_{0} \\
b_{1} \\
b_{2}
\end{array}\right]
$$

The eigenvalues of $\mathrm{A}$ are thus, given by

$$
\begin{aligned}
& \lambda_{1}=-\frac{d_{1}}{2}+i \frac{\sqrt{4 d_{2}-d_{1}^{2}}}{2} \\
& \lambda_{2}=-\frac{d_{1}}{2}-i \frac{\sqrt{4 d_{2}-d_{1}^{2}}}{2}
\end{aligned}
$$

and the eigenvectors are given by

$$
P=\left[\begin{array}{cc}
1 & 1 \\
\lambda_{1} & \lambda_{2}
\end{array}\right]
$$

Having defined the system model and given the initial system state $X_{0}$, the aim is finding the control law and parameters that take the system from the initial state $X_{0}$ to a target state $X_{1}$, minimizing the time to target.

\section{B. Control Law}

The problem of finding the control law that drives the plasma position from an initial position $X_{0}$ to a final position $X_{1}$ in the minimum amount of time, is easier with the definition of a new system state $X_{N}$ and the redefinition of the state system equations. In this state system the set point becomes the origin, thus simplifying the problem:

$$
\begin{aligned}
X_{N} & =X-X_{1} \\
\dot{X}_{N} & =A X_{N}+B u+A X_{1} \\
X_{N 0} & =X_{0}-X_{1}
\end{aligned}
$$
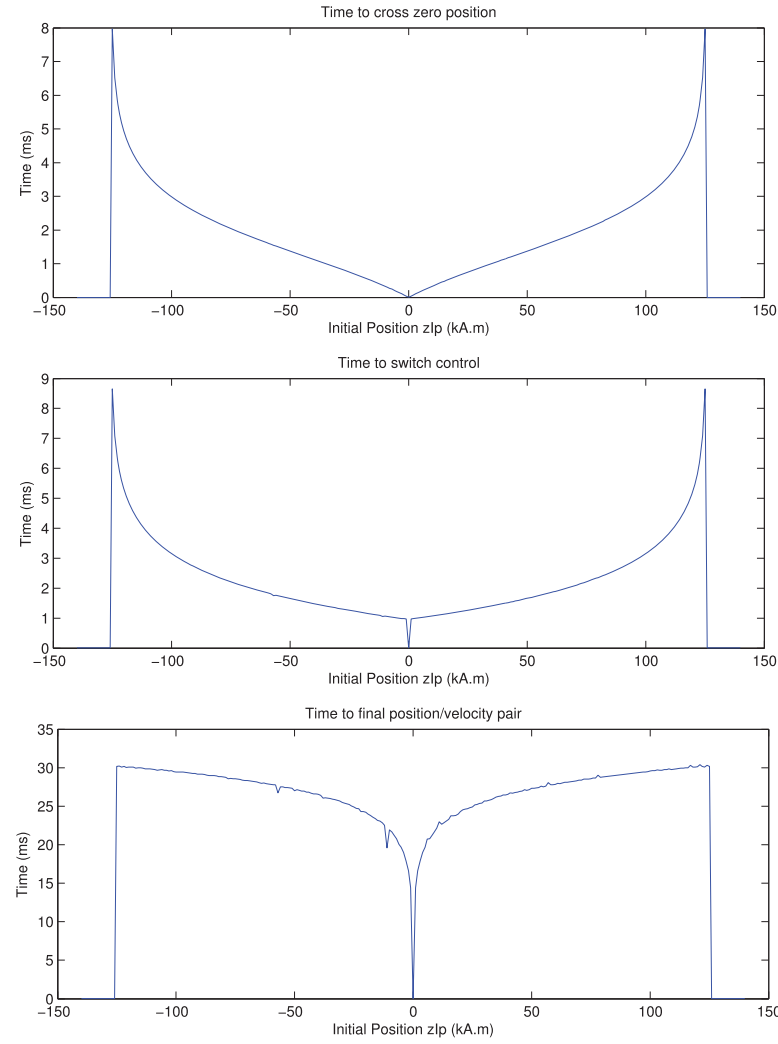

Fig. 3. Representation of the simulated time:(i) to cross the zero position (top); (ii) to switch the control (middle); (iii) to the set point position velocity pair $(0,0)$ (bottom) versus the initial position using the model for discharge 49529.

Using Pontryagin's Minimum Principle (PMP), the aim is to minimize the cost function given by the time to achieve the set point:

$$
J=\int_{0}^{t_{f}} d t
$$

According to PMP the control must minimize the optimal control theory Hamiltonian of the system that is given by:

$$
H=1+\lambda^{T}\left(A X_{N}+B u+A X_{1}\right)
$$

where $\lambda$ is the state of the adjoint system, representing the system as a linear transformation using the vector space defined by the eigenvectors.

The minimization of this Hamiltonian yields the optimal time control law [25]:

$$
\begin{aligned}
& \lambda^{T} B>0 \Rightarrow u=u_{\min } \\
& \lambda^{T} B<0 \Rightarrow u=u_{\max }
\end{aligned}
$$

The bang-bang control law is complete with an arbitrary value of $u$ for $\lambda^{T} B=0$, which might also be a dead zone where no control is applied to avoid unnecessary switching due to hysteresis.

\section{Predictive Control and Construction of Switching Curves}

This subsection presents the method to predict the action ahead, preventing situations when the observer becomes temporarily unavailable, for example in the presence of Edge Localized Modes (ELMs). By the use of this method, it is 


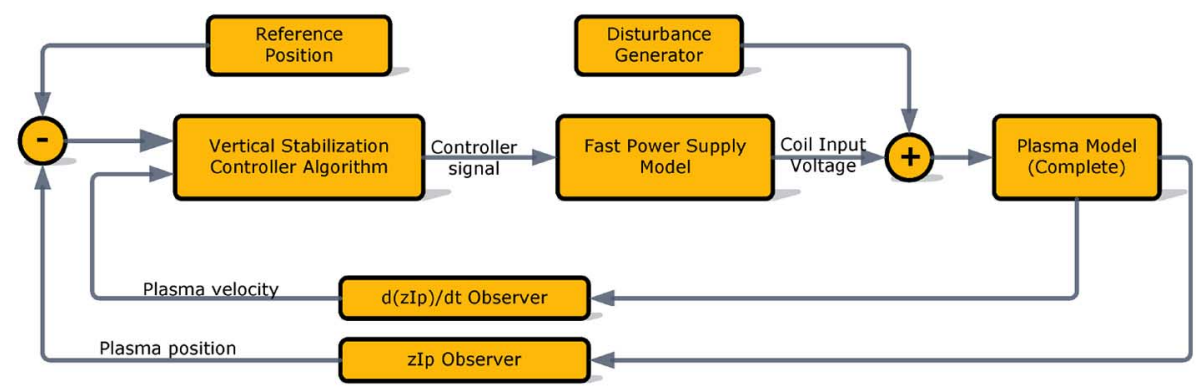

Fig. 4. Block diagram of the simulation tool to analyze controller performance before implementation in real plasma discharges.

possible to keep the system stable, by predicting the control action needed, provided the time the observer is not available is shorter than the final control time calculated and no other major unpredicted disturbance affects the system.

This method is based on the a-priori calculation of the switching time and final time for the optimal time control law of the system. This application uses some of the deduction and results already presented [25], but a new simpler and more generic algorithm was developed. The idea is to find the position where the following two paths cross each other. From the initial system state is applied the maximum control possible in the direction of the set point tracing this path. Also trace the path from the set point applying the opposite control backward in time. The state-space point where both paths cross is the place where the controller should switch. Based on the idea presented the following 5 step fully computational algorithm was developed and implemented:

Step 1) Define what path control $\left(u_{\max } / u_{\min }\right)$ should the system travel first in the direction of the set point, based on the initial system state.

Step 2) Build the trace of the path that the system travels from initial position, when the maximum/minimum control is applied $u_{\max } / u_{\min }$. The path is an array with system state and time information.

Step 3) Build the back trace in time that the system travels, when the maximum/minimum control is applied. This path includes a negative time array that counts the time from $t_{f}$ backward.

Step 4) Calculate the intersection of both paths, leading to the calculation of the desired values. The system state intersection time in the first array gives the switching time $t_{s}$, that can be added to the time in the system state of the second array to give the final time $t_{f}$.

Step 5) Repeat the same procedure to a different initial system state to find a matrix of initial system states versus switching and final times.

\section{Application to the Reduced Plasma Model}

The method of calculation was applied to a set of admissible system states, to drive the system to the desired set point. A table was built, to be implemented in the controller system.

Fig. 3 reproduces the time: (i) to cross the zero position; (ii) to switch the control; and (iii) to the set point position velocity pair $(0,0)$. Moreover, it is possible to check the uncontrollable zones on the left and on the right of the graphics. This method de- fines the controllable zone of the plasma given its initial position and velocity, permitting the simulation of the plasma parameter limits and the design of new control systems.

\section{Controller Simulations}

\section{A. Simulator Tool}

The plasma model was used to build a system simulation tool using Matlab Simulink [26] (Fig. 4). The simulator was implemented to test the different controllers and fine tuning any parameters before the use in real discharges in the tokamak.

The plasma model includes the transfer function between the currents in the internal poloidal field coils and the plasma position, but lacks the transfer function of the fast power supplies that were also taken into account using a different simulation block. The stabilization controller has two inputs: the plasma velocity and plasma position error. From the inputs this block builds the controller signal to be sent to the fast power supplies. A disturbance generator is used to simulate unpredictable influences in the plasma. The complete plasma model is used for the simulation, for accuracy, because there is no need to use a reduced model except for the fact of faster computational simulations. Finally, the plasma model outputs the plasma position and a derivative block is used to simulate the plasma velocity measurements.

This Matlab Simulink model was used to obtain preliminary results.

\section{B. Simulation Analysis}

The controller algorithm was tested and tuned based on simulation analysis. The decision for the best controller based on these analyses, resulted in a controller that adapts its force to the initial velocity detected.

A true bang-bang controller that always applies the maximum restore signal would exhibit a big oscillation in the plasma position. On the opposite side, a bang-bang controller that was limited to use a small control signal avoiding to exhibit oscillations, would be limited to the control of small perturbations. Thus, a weighted bang-bang controller that increases its restoring signal according to the initial plasma velocity demonstrated to be much more efficient, resulting in a more stable controller.

Fig. 5 supports the use of a weighted bang-bang controller.

In these simulations it is possible to see a bang-bang controller with maximum possible strength that was tested against a high level of disturbances (Fig. 5 on the left) with the plasma position under good control. However, using a variable bang-bang controller that changes state according to the distance of the 

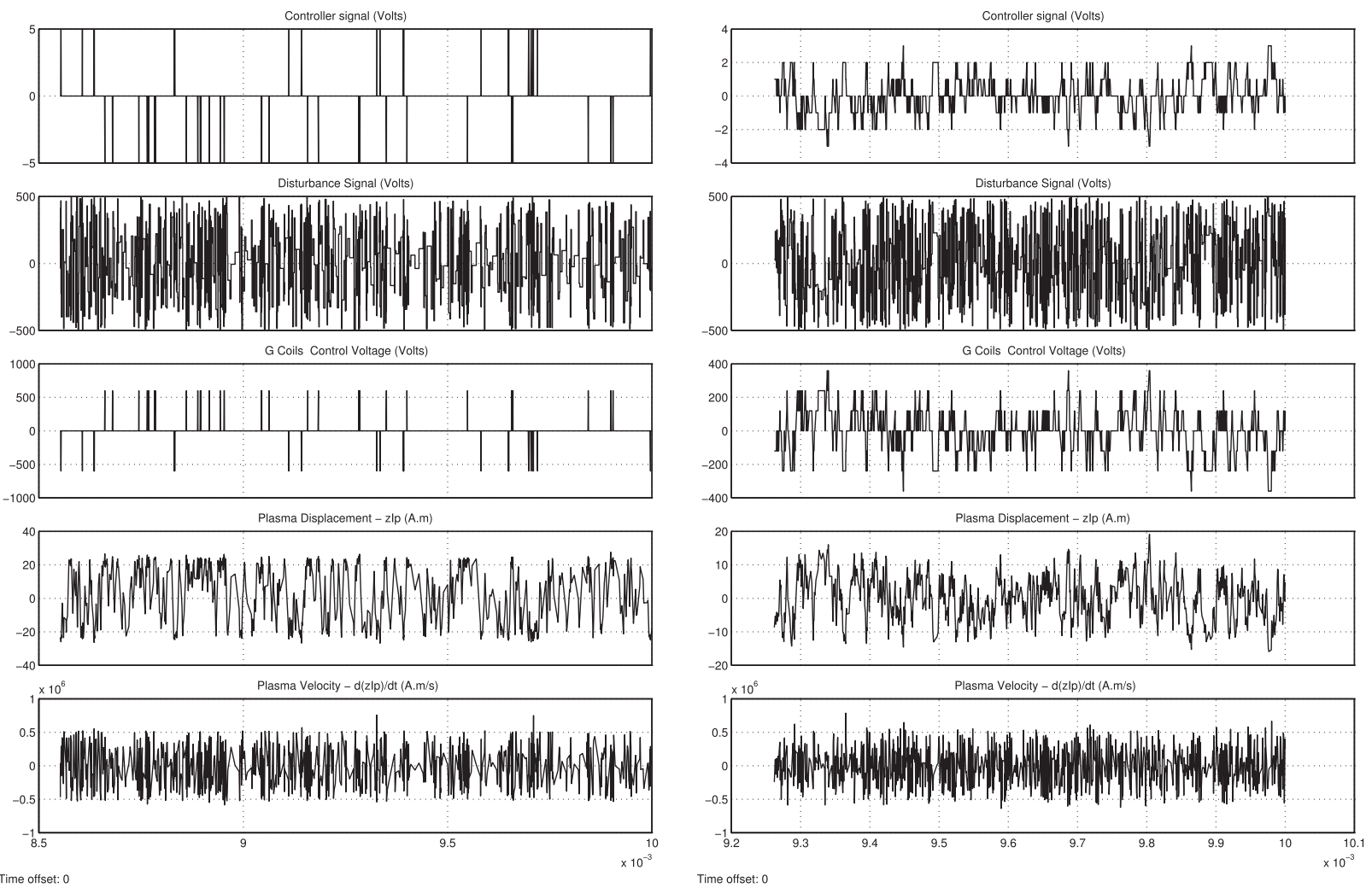

Time offet:0

Fig. 5. Simulation results of the bang-bang (left) and variable bang-bang (right) controllers.

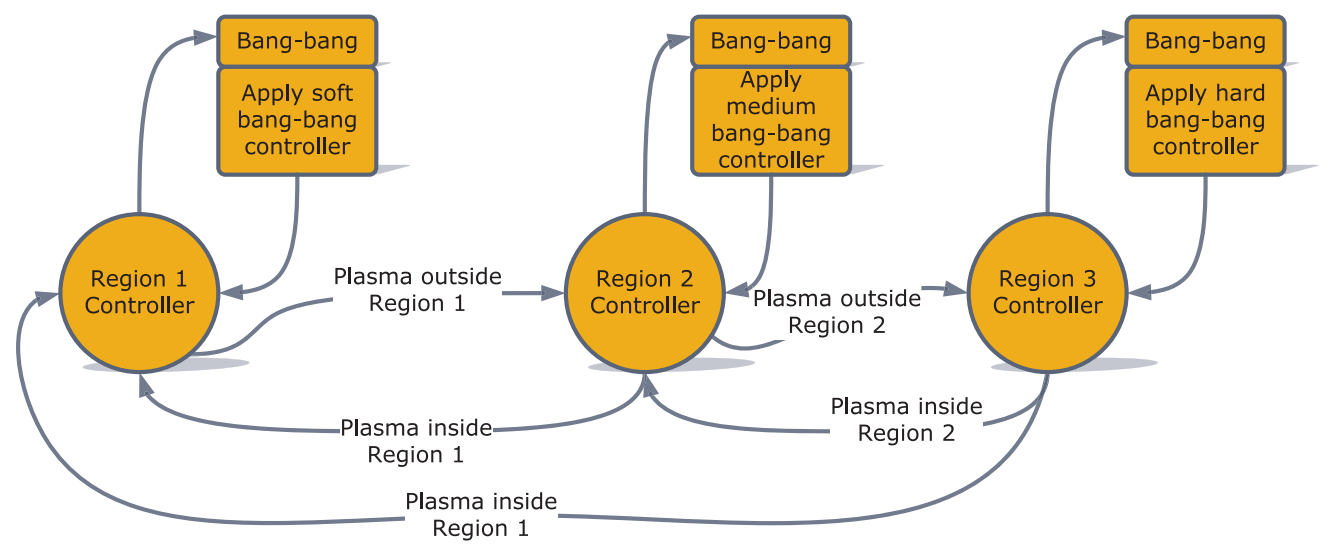

Fig. 6. Diagram of the controller state machine.

plasma to the set point (Fig. 5 on the right), also in the presence of big disturbances, the coil currents needed to stabilize the plasma are lower, as well as the plasma position error. The analysis of further simulations show that big disturbances can be controlled using a high control signal for higher displacements and smaller control signal for smaller displacements.

Fig. 6 represents a diagram with the controller state-machine. The controller is a weighted bang-bang controller, that is similar to use an adaptive bang-bang controller that reconfigures based on system state position and velocity limits. This controller option improves stability by introducing a linear component to the classical nonlinear bang-bang controller.

\section{CONTROller VALidation AND Results}

The controller implementation based on the simulation results was tested during plasma discharges at TCV, with improvement in the overall stability of the plasma. Figs. 7 and 8 depict the stability improvement using the new controller. The plasma discharges were designed to test the limits of the controllers by increasing the plasma elongation from 0.5 seconds, which can be seen at the top of each figure. These plots already confirm the higher elongation achieved using the bang-bang controller.

The increased instability limit using the new controller can also be confirmed by the improvement in discharge time for the same conditions. The current PID controller was not able to cope with the vertical instability finishing the discharge with a vertical disruption at approximately $0.65 \mathrm{~s}(0.15 \mathrm{~s}$ after starting the linear increase in plasma elongation). On the other hand the new bang-bang controller maintained the plasma discharge up to approximately $0.8 \mathrm{~s}(0.3 \mathrm{~s}$ after starting the linear increase in plasma elongation).

Figs. 7 and 8 also show a smaller deviation for the plasma position and velocity during the discharge. Figs. 9 and 10 depict a 

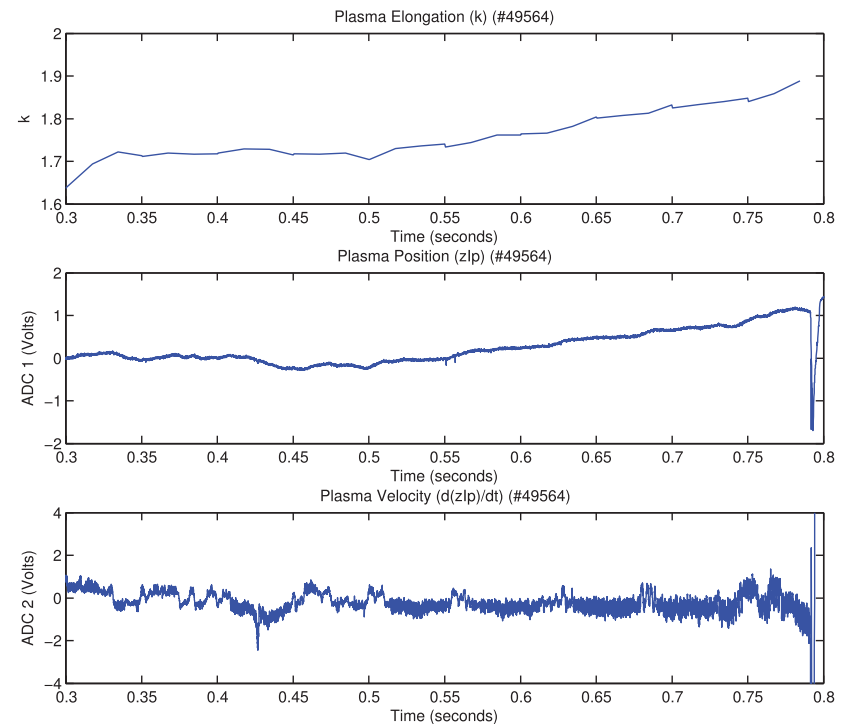

Fig. 7. Plasma position and velocity for shot 49564 using the new bang-bang controller.
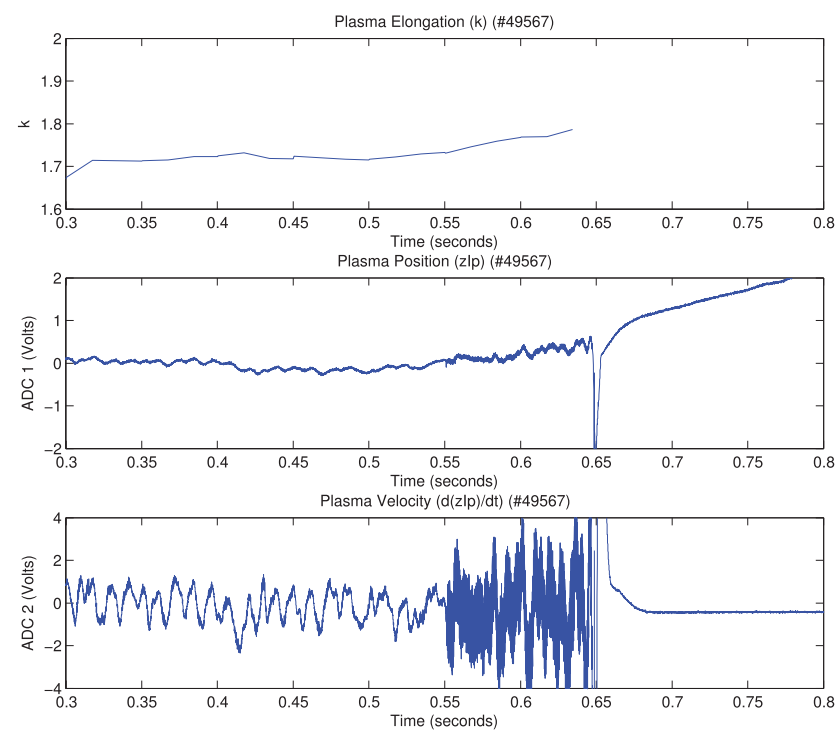

Fig. 8. Plasma position and velocity for reference shot 49567 using the PID controller in the same plasma conditions as discharge 49564.

better use of the coil currents. The plasma position and velocity are more stable during the complete discharge without the continuous fast up-down movement that can be seen using the PID controller.

In conclusion, the stabilization of the axisymmetric MHD instability of highly elongated plasmas requires very fast responses that can not be provided by reconstruction algorithms, some of which presented in Section III. The internal stabilization coils of TCV are designed to counter growth rates of up to $3000 \mathrm{~s}^{-1}$. The bang-bang controller developed was an effort to improve even further over the performance of the analogue Plant Control System (PCS). This was implemented in the APCS digital signal processors and tested successfully on TCV discharges featuring an elongation ramp: the higher elongation achieved by the new bang-bang controller than by the standard PID one will be exploited to improve the TCV plasma stability during the future campaigns of TCV Tokamak Science Program [27].
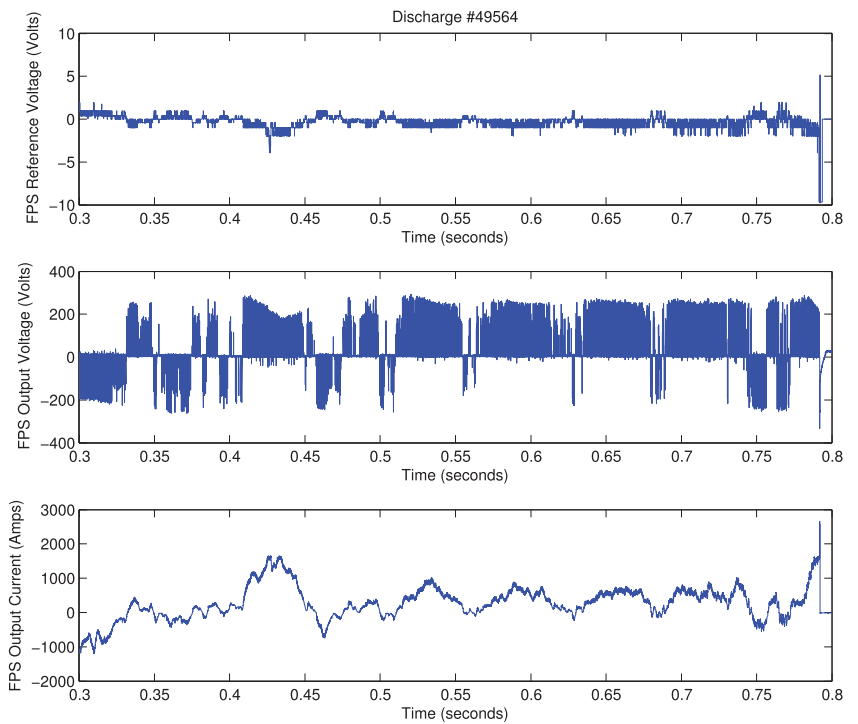

Fig. 9. Control signal and coil current for discharge 49564 using the new bangbang controller.
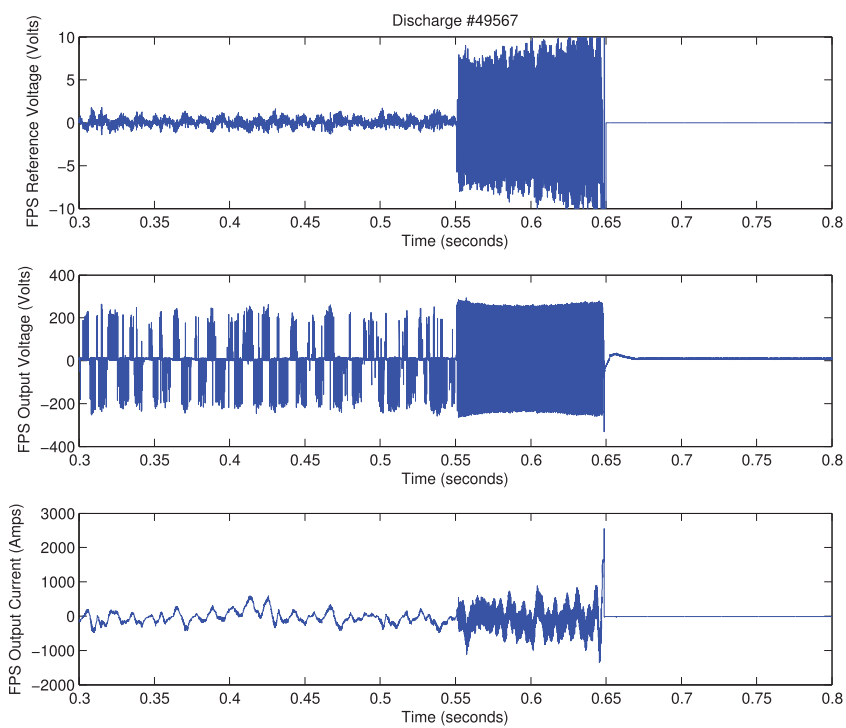

Fig. 10. Control signal and coil current for reference discharge 49567 using the PID controller in the same plasma conditions as discharge 49564.

The vertical stabilization controller was implemented and tested using one of the hardware modules with parallel digital signal processing capabilities of the Advanced Plasma Control System [28]. For further testing of the controller it is envisaged the use of an ELM detector [29] capable of signalling the error and unavailability of plasma position observer. It is also planned the controller implementation in a newer control hardware based on FPGA [30] to study and compare the performance of both systems.

\section{REFERENCES}

[1] J. Wesson, Tokamaks. Oxford, U.K.: Oxford Univ. Press, 2004.

[2] M. Ariola and A. Pironti, Magnetic control of tokamak plasmas. New York, NY, USA: Springer-Verlag, 2008.

[3] G. Ambrosino and R. Albanese, "Magnetic control of plasma current, position, and shape in tokamaks - A survey of modeling and control approaches," IEEE Control Syst. Mag., vol. 25, no. 5, pp. 76-92, Oct. 2005. 
[4] F. Sartori, A. Barbalace, A. J. N. Batista, T. Bellizio, P. Card, G. De Tommasi, P. Mc Cullen, A. Neto, F. Piccolo, R. Vitelli, and L. Zabeo, "JET-EFDA contributors, the PCU JET plasma vertical stabilization control system, fusion engineering and design," in Proc. 7th IAEA Tech. Meeting Control, Data Acquisit., Remote Participat. for Fusion Res., Jul. 2010, vol. 85, no. 3-4, pp. 438-442.

[5] P. Vyas, D. Mustafa, and A. W. Morris, "Vertical position control on COMPASS-D," Fusion Technol., vol. 33, no. 2, pp. 97-105, 1998.

[6] M. L. Walker and D. A. Humphreys, "On feedback stabilization of the tokamak plasma vertical instability," Automatica, vol. 45, no. 3, pp. 665-674, Mar. 2009.

[7] G. Ambrosino, M. Ariola, G. D. Tommasi, and A. Pironti, "Plasma vertical stabilization in the ITER tokamak via constrained static output feedback," IEEE Trans. Control Syst. Technnol., vol. 19, pp. 376-381, Mar. 2011.

[8] M. Ariola, G. De Tommasi, A. Pironti, and F. Villone, "Control of resistive wall modes in tokamak plasmas," Control Eng. Practice, vol. 24, pp. 15-24, Mar. 2014.

[9] F. Hofmann, M. J. Dutch, A. Favre, Y. Martin, J.-M. Moret, and D. J. Ward, "Feedback stabilization of axisymmetric modes in the TCV tokamak using active coils inside and outside the vacuum vessel," Nucl. Fusion, vol. 38, pp. 399-408, 1998.

[10] J.-M. Moret, B. P. Duval, H. B. Le, S. Coda, F. Felici, and H. Reimerdes, "Tokamak equilibrium reconstruction code LIUQE and its real time implementation," Fusion Eng. Design, Jul. 28, 2014, accepted for publication.

[11] N. Cruz, J.-M. Moret, S. Coda, J. I. Paley, B. P. Duval, A. P. Rodrigues, F. Piras, F. Felici, C. M. B. A. Correia, and C. A. F. Varandas, "Using APCS for plasma vertical control at TCV," IEEE Trans. Nucl. Science, vol. 58, no. 4, pp. 1570-1575, Aug. 2011.

[12] S. A. Galkin, A. A. Ivanov, S. Y. Medvedev, and Y. Y. Poshekhonov, "Comparison of tokamak axisymmetric mode growth rates from linear MHD and equilibrium evolution approaches," Nucl. Fusion, vol. 37, pp. 1455-1461, 1997.

[13] S. C. Jardin, N. Pomphrey, and J. De Lucia, "Dynamic modeling of transport and positional control of tokamaks," J. Comput. Phys., vol. 66, pp. 481-507, 1986.

[14] L. L. Lao, J. R. Ferron, R. J. Groebner, W. Howl, H. St. John, E. J. Strait, and T. S. Taylor, "Equilibrium analysis of current profiles in tokamaks," Nucl. Fusion, vol. 30, pp. 1035-1050, 1990.

[15] F. Hofmann, "FBT - a free-boundary tokamak equilibrium code for highly elongated and shaped plasmas," Comput. Phys. Commun., vol. 48, no. 2, pp. 207-221, 1988.

[16] R. Albanese, J. Blum, and O. De Barbieri, "Numerical studies of the NEXT European torus via the proteus code," in Proc. 12th Conf. Numer. Simulat. Plasmas, San Francisco, CA, USA, 1987.
[17] R. Albanese and F. Villone, "The linearized CREATE-L plasma response model for the control of current, position and shape in tokamaks," Nucl. Fusion, vol. 38, pp. 723-738, 1998.

[18] R. Khayrutdinov, "Studies of plasma equilibrium and transport in a tokamak fusion device with the inverse-variable technique," $J$. Comput. Phys., vol. 109, pp. 193-201, 1993.

[19] A. Coutlis, I. Bandyopadhyay, J. B. Lister, P. Vyas, R. Albanese, D. J. N. Limebeer, F. Villone, and J. P. Wainwright, "Measurement of the open loop plasma equilibrium response in TCV," Nucl. Fusion, vol. 39, pp. 663-683, 1999.

[20] E. A. Lazarus, J. B. Lister, and G. H. Neilson, "Control of the vertical instability in tokamaks," Nucl. Fusion, vol. 30, no. 1, pp. 111-141, 1990.

[21] F. Hofmann et al., "Vertical instability in TCV: Comparison of experimental and theoretical growth rates," Nucl. Fusion, vol. 37, pp. 681-687, 1997.

[22] A. J. Laub, M. T. Heath, C. C. Paige, and R. C. Ward, "Computation of system balancing transformations and other applications of simultaneous diagonalization algorithms," IEEE Trans. Autom. Control, vol. AC-32, pp. 115-122, Feb. 1987.

[23] M. Athans and P. L. Falb, Optimal Control: An Introduction to the Theory and its Applications. New York, NY, USA: McGraw-Hill, 1966.

[24] I. Vakilzadeh and A. A. Keshavarz, "Bang-bang control of a secondorder non-linear stable plant with second-order nonlinearity," Kybernetika, vol. 18, no. 1, pp. 67-71, 1982.

[25] Z. Shen and S. B. Andersson, "Minimum time control of a secondorder system," in Proc. 49th IEEE Conf. Decision Control, Atlanta, GA, USA, Dec. 15-17, 2010, pp. 4819-4824.

[26] [Online]. Available: www.mathworks.com/products/simulink/

[27] S. Coda, for the TCV team, "The science program of the TCV tokamak: Exploring fusion reactor and power plant concepts," in Proc. 25th IAEA FEC - Fusion Energy Conf., St-Petersburg, Russia, Oct. 2014.

[28] N. Cruz, A. P. Rodrigues, B. Santos, C. A. F. Varandas, B. P. Duval, J.-M. Moret, J. Berrino, Y. Martin, and X. Llobet, "The integration of the new advanced digital plasma control system in TCV," Fusion Eng. Design, vol. 83, pp. 215-219.

[29] F. Felici, J. X. Rossel, B. P. Duval, S. Coda, T. P. Goodman, Y. Martin, J.-M. Moret, and O. Sauter, for the TCV team, "Real-time control of the period of individual ELMs by EC power on TCV," Nucl. Fusion, vol. 53, p. 113018, 2013.

[30] H. B. Le, B. P. Duval, J.-M. Moret, and N. Cruz, and the TCV team, "New developments for real time plasma control system of tcv tokamak based on FPGA," in Proc. 19th IEEE-NPSS Real Time Conf., Nara, Japan, May 2014. 